\title{
Association of a polymorphism of BTN2A1 with chronic kidney disease in individuals with or without hypertension or diabetes mellitus
}

\author{
TETSURO YOSHIDA ${ }^{1}$, KIMIHIKO KATO ${ }^{2}$, MITSUTOSHI OGURI ${ }^{3}$, HIDEKI HORIBE ${ }^{5}$, \\ TOSHIKI KAWAMIYA ${ }^{5}$, KIYOSHI YOKOI ${ }^{5}$, TETSUO FUJIMAKI ${ }^{6}$, SACHIRO WATANABE ${ }^{7}$, \\ KEI SATOH ${ }^{8}$, YUKITOSHI AOYAGI ${ }^{9}$, MASASHI TANAKA $^{9}$, HIROTO YOSHIDA $^{10}$, \\ SHOJI SHINKAI ${ }^{10}$, YOSHINORI NOZAWA ${ }^{11}$ and YOSHIJI YAMADA ${ }^{4}$
}

\begin{abstract}
${ }^{1}$ Department of Cardiovascular Medicine, Onga Nakama Medical Association Onga Hospital, Onga;
${ }^{2}$ Meitoh Hospital; ${ }^{3}$ Department of Cardiology, Japanese Red Cross Nagoya First Hospital, Nagoya;

${ }^{4}$ Department of Human Functional Genomics, Life Science Research Center, Mie University, Tsu;

${ }^{5}$ Department of Cardiovascular Medicine, Gifu Prefectural Tajimi Hospital, Tajimi;

${ }^{6}$ Department of Cardiovascular Medicine, Inabe General Hospital, Inabe; ${ }^{7}$ Department of Cardiology,

Gifu Prefectural General Medical Center, Gifu; ${ }^{8}$ Department of Vascular Biology, Institute of Brain Science, Hirosaki University Graduate School of Medicine, Hirosaki; ${ }^{9}$ Department of Genomics for Longevity and Health, and ${ }^{10}$ Research Team for Social Participation and Health Promotion, Tokyo Metropolitan Institute of Gerontology,

Tokyo; ${ }^{11}$ Gifu International Institute of Biotechnology and Tokai Gakuin University, Kakamigahara, Japan
\end{abstract}

Received December 14, 2010; Accepted January 4, 2011

DOI: $10.3892 /$ etm.2011.191

\begin{abstract}
Hypertension and diabetes mellitus are important risk factors for chronic kidney disease (CKD). We previously showed that the $\mathrm{C} \rightarrow \mathrm{T}$ polymorphism (rs6929846) of BTN2Al was significantly associated with myocardial infarction. The purpose of the present study was to examine an association of rs6929846 of BTN2Al with CKD in individuals with or without hypertension or diabetes mellitus, thereby contributing to the personalized prevention of CKD in such individuals separately. The study population comprised 7,542 unrelated individuals, including 2,289 subjects with CKD [estimated glomerular filtration rate $(\mathrm{eGFR})<60 \mathrm{ml} / \mathrm{min} / 1.73 \mathrm{~m}^{2}$ ] and 5,253 controls (eGFR $\geq 60 \mathrm{ml} / \mathrm{min} / 1.73 \mathrm{~m}^{2}$ ) with or without hypertension or diabetes mellitus. The Chi-square test, a multivariable logistic regression analysis with adjustment for covariates, as well as a stepwise forward selection procedure revealed that the $\mathrm{C} \rightarrow \mathrm{T}$ polymorphism (rs6929846) of BTN2Al was significantly associated with $\mathrm{CKD}$ in normotensive individuals, in diabetic individuals and in individuals with hypertension and diabetes mellitus, or without either condition, with the $T$ allele representing a risk factor for
\end{abstract}

Correspondence to: Dr Yoshiji Yamada, Department of Human Functional Genomics, Life Science Research Center, Mie University, 1577 Kurima-machiya, Tsu, Mie 514-8507, Japan

E-mail: yamada@gene.mie-u.ac.jp

Key words: genetics, polymorphism, chronic kidney disease, diabetes mellitus, hypertension
CKD. Stratification of subjects based on hypertension or diabetes mellitus may thus be important in order to achieve personalized prevention of CKD with the use of genetic information.

\section{Introduction}

Chronic kidney disease (CKD) is an important global public health problem because of its high morbidity in the general population. Recent studies suggest that the risk for death is increased in individuals who have impaired renal function, but do not require dialysis, compared to those who have preserved renal function $(1,2)$. Disease prevention is an important strategy for reducing the overall burden of CKD and end-stage renal disease (ESRD), and the identification of markers for CKD risk is essential both for risk prediction and for potential intervention to reduce the chance of future cardiovascular complications (3).

Several risk factors for progression of CKD have been proposed (4), with hypertension and diabetes mellitus having been recognized as important risk factors for CKD (5-7) as well as for coronary heart disease and stroke. In addition to these conventional risk factors, recent studies have shown the importance of genetic factors and of interactions between multiple genes and environmental factors in the development of CKD $(8,9)$. Although recent genome-wide association studies (GWASs) (10-15) have implicated several loci and candidate genes in predisposition to CKD or ESRD in Caucasians or African-American populations, the genes that contribute to genetic susceptibility to CKD in Asian populations remain to be identified definitively. 
We previously showed that the $\mathrm{C} \rightarrow \mathrm{T}$ polymorphism (rs6929846) of BTN2Al was significantly associated with myocardial infarction in Japanese individuals by a GWAS (16). Given that CKD is an important risk factor for coronary heart disease, the association of rs6929846 with myocardial infarction may be attributable, at least in part, to its effect on susceptibility to CKD. We also showed that genetic variants that confer susceptibility to CKD differ between individuals with or without metabolic syndrome (17), between individuals with or without type 2 diabetes mellitus (18), between individuals with or without hypertension (8), or between individuals with different lipid profiles (19). To further examine whether the association of a genetic variant with CKD is influenced by the absence or presence of hypertension or diabetes mellitus, we performed an association study for rs6929846 of BTN2A1 and CKD in 7,542 Japanese individuals with or without hypertension or diabetes mellitus.

\section{Materials and methods}

Study population. The study population comprised 7,542 unrelated Japanese individuals (4,382 men and 3,160 women) who either visited the outpatient clinics of or were admitted to the participating hospitals (Gifu Prefectural General Medical Center, Gifu; Gifu Prefectural Tajimi Hospital, Tajimi; Hirosaki University Hospital and Hirosaki Stroke Center, Hirosaki; Japanese Red Cross Nagoya First Hospital, Nagoya; and Inabe General Hospital, Inabe, Japan) between October 2002 and March 2009 because of various symptoms or for an annual health checkup, or who were recruited to populationbased prospective cohort studies of aging and age-related diseases in Nakanojo, Kusatsu and Tokyo, Japan.

Glomerular filtration rate was estimated with the use of the simplified prediction equation derived from that in the Modification of Diet in Renal Disease Study and proposed by the Japanese Society of Nephrology (20): eGFR (ml/ $\left.\min / 1.73 \mathrm{~m}^{2}\right)=194 \mathrm{x}$ [age (years) $]^{-0.287} \times$ [serum creatinine $(\mathrm{mg} / \mathrm{dl})]^{-1.094}[\mathrm{x} \quad 0.739$ if female]. The National Kidney Foundation-Kidney Disease Outcomes Quality Initiative guidelines recommend a diagnosis of CKD if eGFR is $<60 \mathrm{ml} /$ $\min / 1.73 \mathrm{~m}^{2}(3)$. Non-linear relations between GFR and the risk of adverse outcomes, such as death, cardiovascular events and hospitalization, have been demonstrated, with an increased risk being associated with an eGFR of $<60 \mathrm{ml} / \mathrm{min} / 1.73 \mathrm{~m}^{2}$ (1). We thus adopted the criterion of an eGFR of $<60 \mathrm{ml} /$ $\min / 1.73 \mathrm{~m}^{2}$ for the diagnosis of CKD in the present study. On the basis of this criterion, 2,289 subjects (1,394 men and 895 women) were diagnosed with CKD. The control subjects comprised 5,253 individuals (2,988 men and 2,265 women) whose eGFR was $\geq 60 \mathrm{ml} / \mathrm{min} / 1.73 \mathrm{~m}^{2}$.

Subjects with CKD and controls either had or did not have conventional risk factors for CKD, including hypertension (systolic blood pressure of $\geq 140 \mathrm{mmHg}$, diastolic blood pressure of $\geq 90 \mathrm{mmHg}$, or taking of antihypertensive medication), diabetes mellitus [fasting plasma glucose level of $\geq 6.93 \mathrm{mmol} / \mathrm{l}$, blood glycosylated hemoglobin (HbAlc) content of $\geq 6.5 \%$, or taking of antidiabetes medication], or hypercholesterolemia (serum total cholesterol concentration of $\geq 5.72 \mathrm{mmol} / 1$, or taking of lipid-lowering medication). The study protocol complied with the Declaration of Helsinki and was approved by the Committees on the Ethics of Human Research of Mie University Graduate School of Medicine, Hirosaki University Graduate School of Medicine, Gifu International Institute of Biotechnology, Tokyo Metropolitan Institute of Gerontology and participating hospitals. Written informed consent was obtained from each participant.

Genotyping of rs6929846 single nucleotide polymorphism $(S N P)$. Venous blood $(7 \mathrm{ml})$ was collected into tubes containing $50 \mathrm{mmol} / \mathrm{l}$ ethylenediaminetetraacetic acid (disodium salt), and genomic DNA was isolated with a kit (Genomix; Talent, Trieste, Italy). Genotypes of rs6929846 of BTN2Al were determined at G\&G Science (Fukushima, Japan) by a method that combines the polymerase chain reaction (PCR) and sequence-specific oligonucleotide probes with suspension array technology (Luminex, Austin, TX, USA). Primers, probes and other PCR conditions for the genotyping of rs6929846 have been described previously (16).

Statistical analysis. Quantitative data were compared between subjects with CKD and controls by the unpaired Student's t-test. Categorical data were compared by the Chi-square test. Allele frequencies were estimated by the gene counting method, and the Chi-square test was used to identify departures from Hardy-Weinberg equilibrium. Genotype distribution of rs6929846 was compared between subjects with CKD and controls by the Chi-square test. When a P-value for genotype distribution was $<0.05$, multivariable logistic regression analysis was performed with adjustment for covariates. Multivariable logistic regression analysis was thus performed with CKD as a dependent variable, and independent variables, including age, gender (0, woman; 1 , man), body mass index (BMI), smoking status (0, non-smoker; 1 , smoker), history of hypertension, diabetes mellitus or hypercholesterolemia $(0$, no history; 1, positive history) and genotype of rs6929846. P-values, odds ratios and $95 \%$ confidence intervals were calculated. Genotype was assessed according to dominant, recessive and additive genetic models. Additive models included the additive 1 (heterozygotes vs. wild-type homozygotes) and additive 2 (variant homozygotes vs. wild-type homozygotes) models, which were analyzed simultaneously with a single statistical model. We also performed a stepwise forward selection procedure to examine the effects of genotype as well as of other covariates on CKD; genotype was examined according to a dominant model on the basis of statistical significance in the multivariable logistic regression analysis. The P-levels for inclusion in and exclusion from the model were 0.25 and 0.1 , respectively. A P-value of $<0.05$ was considered statistically significant. Statistical significance was examined by two-sided tests performed with JMP version 6.0 and JMP Genomics version 3.2 software (SAS Institute, Cary, NC, USA).

\section{Results}

Characteristics of subjects. The characteristics of the subjects are shown in Tables I-III. In all analyses, serum concentrations of creatinine were greater and eGFRs were lower in subjects with CKD than in controls. For hypertensive individuals, age and the prevalence of diabetes mellitus were greater, whereas the percentage of smokers and diastolic blood pressure were 
Table I. Characteristics of subjects with chronic kidney disease (CKD) and controls among individuals with or without hypertension.

\begin{tabular}{|c|c|c|c|c|c|c|}
\hline \multirow[b]{2}{*}{ Characteristic } & \multicolumn{3}{|c|}{ With hypertension } & \multicolumn{3}{|c|}{ Without hypertension } \\
\hline & CKD & Controls & P-value & CKD & Controls & P-value \\
\hline No. of subjects & 1,662 & 3,241 & & 627 & 2,012 & \\
\hline Age (years) & $71.0 \pm 8.7$ & $66.9 \pm 9.7$ & $<0.0001$ & $70.6 \pm 9.4$ & $63.8 \pm 11.0$ & $<0.0001$ \\
\hline Gender (male/female, \%) & $62.2 / 37.8$ & $60.8 / 39.2$ & 0.3409 & $57.4 / 42.6$ & $50.6 / 49.4$ & 0.0026 \\
\hline Body mass index $\left(\mathrm{kg} / \mathrm{m}^{2}\right)$ & $23.7 \pm 3.4$ & $23.8 \pm 3.4$ & 0.1455 & $23.1 \pm 3.3$ & $23.0 \pm 3.2$ & 0.3631 \\
\hline Current or former smoker $(\%)$ & 26.3 & 31.4 & 0.0002 & 27.3 & 27.9 & 0.7655 \\
\hline Systolic blood pressure (mmHg) & $150 \pm 25$ & $149 \pm 23$ & 0.5850 & $121 \pm 12$ & $122 \pm 12$ & 0.3656 \\
\hline Diastolic blood pressure (mmHg) & $80 \pm 15$ & $82 \pm 14$ & $<0.0001$ & $70 \pm 10$ & $71 \pm 9$ & 0.0156 \\
\hline Diabetes mellitus (\%) & 43.5 & 40.1 & 0.0237 & 28.4 & 24.5 & 0.0530 \\
\hline Hypercholesterolemia (\%) & 27.7 & 27.2 & 0.7131 & 27.1 & 28.2 & 0.5854 \\
\hline Serum creatinine $(\mu \mathrm{mol} / \mathrm{l})$ & $118.9 \pm 131.5$ & $62.4 \pm 12.5$ & $<0.0001$ & $90.7 \pm 23.5$ & $61.5 \pm 12.2$ & $<0.0001$ \\
\hline eGFR (ml/min/1.73 m²) & $47.5 \pm 11.8$ & $78.9 \pm 15.6$ & $<0.0001$ & $51.1 \pm 7.5$ & $79.0 \pm 17.9$ & $<0.0001$ \\
\hline
\end{tabular}

Quantitative data are the means \pm SD. eGFR, estimated glomerular filtration rate.

Table II. Characteristics of subjects with chronic kidney disease (CKD) and controls among individuals with or without diabetes mellitus.

\begin{tabular}{|c|c|c|c|c|c|c|}
\hline \multirow[b]{2}{*}{ Characteristic } & \multicolumn{3}{|c|}{ With diabetes mellitus } & \multicolumn{3}{|c|}{ Without diabetes mellitus } \\
\hline & CKD & Controls & $\mathrm{P}$-value & CKD & Controls & P-value \\
\hline No. of subjects & 901 & 1,794 & & 1,388 & 3,459 & \\
\hline Age (years) & $70.1 \pm 9.2$ & $66.9 \pm 9.6$ & $<0.0001$ & $71.3 \pm 8.7$ & $65.1 \pm 10.7$ & $<0.0001$ \\
\hline Gender (male/female, \%) & $66.3 / 33.7$ & $65.7 / 34.3$ & 0.7799 & $57.4 / 42.6$ & $52.3 / 47.7$ & 0.0012 \\
\hline Body mass index $\left(\mathrm{kg} / \mathrm{m}^{2}\right)$ & $23.9 \pm 3.6$ & $23.9 \pm 3.5$ & 0.8711 & $23.3 \pm 3.3$ & $23.3 \pm 3.2$ & 0.8712 \\
\hline Current or former smoker $(\%)$ & 27.3 & 33.4 & 0.0011 & 26.1 & 28.3 & 0.1120 \\
\hline Hypertension (\%) & 80.2 & 72.5 & $<0.0001$ & 67.7 & 56.1 & $<0.0001$ \\
\hline Fasting plasma glucose $(\mathrm{mmol} / \mathrm{l})$ & $9.08 \pm 3.68$ & $9.15 \pm 3.62$ & 0.4282 & $5.36 \pm 0.84$ & $5.30 \pm 0.84$ & 0.0139 \\
\hline Blood glycosylated hemoglobin (\%) & $6.67 \pm 1.76$ & $6.65 \pm 1.76$ & 0.4595 & $5.25 \pm 0.35$ & $5.23 \pm 0.38$ & 0.0288 \\
\hline Hypercholesterolemia (\%) & 26.2 & 27.7 & 0.4049 & 28.4 & 27.5 & 0.5444 \\
\hline Serum creatinine $(\mu \mathrm{mol} / \mathrm{l})$ & $118.6 \pm 114.2$ & $62.3 \pm 12.6$ & $<0.0001$ & $106.3 \pm 112.7$ & $62.0 \pm 12.2$ & $<0.0001$ \\
\hline eGFR (ml/min/1.73 m²) & $46.9 \pm 11.8$ & $80.5 \pm 16.6$ & $<0.0001$ & $49.5 \pm 10.2$ & $78.1 \pm 16.4$ & $<0.0001$ \\
\hline
\end{tabular}

Quantitative data are the means \pm SD. eGFR, estimated glomerular filtration rate.

lower in subjects with CKD than in controls. For normotensive individuals, age and the frequency of male subjects were greater, whereas diastolic blood pressure was lower in subjects with CKD than in controls (Table I).

For diabetic individuals, age and the prevalence of hypertension were greater, whereas the percentage of smokers was lower in subjects with CKD than in controls. For non-diabetic individuals, age, the frequency of male subjects, the prevalence of hypertension, fasting plasma glucose level and blood glycosylated hemoglobin content were greater in subjects with CKD than in controls (Table II).

For individuals with hypertension and diabetes mellitus, age was greater, whereas the percentage of smokers and diastolic blood pressure was lower in subjects with CKD than in controls. For individuals without hypertension or diabetes mellitus, age and the frequency of male subjects were greater in subjects with CKD than in controls (Table III).

Association of rs6929846 of BTN2A1 with CKD. Comparison of genotype distributions by the Chi-square test revealed that the $\mathrm{C} \rightarrow \mathrm{T}$ polymorphism (rs6929846) of BTN2Al was significantly $(\mathrm{P}<0.05)$ associated with $\mathrm{CKD}$ in normotensive individuals, in diabetic individuals and in individuals with hypertension and diabetes mellitus, or without either condition (Table IV).

Multivariable logistic regression analysis with adjustment for age, gender, BMI, smoking status and the prevalence of hypertension, diabetes mellitus or hypercholesterolemia revealed that rs6929846 of BTN2Al was significantly associated with CKD in normotensive individuals (dominant 
Table III. Characteristics of subjects with chronic kidney disease (CKD) and controls among individuals with hypertension and diabetes mellitus, or without either condition.

\begin{tabular}{|c|c|c|c|c|c|c|}
\hline \multirow[b]{2}{*}{ Characteristic } & \multicolumn{3}{|c|}{$\begin{array}{l}\text { With hypertension and } \\
\text { diabetes mellitus }\end{array}$} & \multicolumn{3}{|c|}{$\begin{array}{l}\text { Without hypertension or } \\
\text { diabetes mellitus }\end{array}$} \\
\hline & CKD & Controls & P-value & CKD & Controls & P-value \\
\hline No. of subjects & 723 & 1,301 & & 449 & 1,519 & \\
\hline Age (years) & $70.1 \pm 9.0$ & $67.2 \pm 9.4$ & $<0.0001$ & $70.6 \pm 9.1$ & $63.1 \pm 11.3$ & $<0.0001$ \\
\hline Gender (male/female, \%) & $66.5 / 33.5$ & $65.9 / 34.1$ & 0.7650 & $54.3 / 45.7$ & $45.7 / 54.3$ & 0.0014 \\
\hline Body mass index $\left(\mathrm{kg} / \mathrm{m}^{2}\right)$ & $24.0 \pm 3.6$ & $24.1 \pm 3.5$ & 0.2262 & $23.0 \pm 3.2$ & $22.9 \pm 3.1$ & 0.7429 \\
\hline Current or former smoker $(\%)$ & 26.7 & 33.1 & 0.0025 & 26.3 & 25.8 & 0.8405 \\
\hline Systolic blood pressure (mmHg) & $152 \pm 26$ & $150 \pm 23$ & 0.4352 & $121 \pm 12$ & $122 \pm 12$ & 0.3550 \\
\hline Diastolic blood pressure (mmHg) & $80 \pm 15$ & $82 \pm 15$ & 0.0047 & $70 \pm 10$ & $72 \pm 9$ & 0.0637 \\
\hline Fasting plasma glucose (mmol/1) & $9.03 \pm 3.58$ & $9.19 \pm 3.59$ & 0.1511 & $5.22 \pm 0.85$ & $5.16 \pm 0.85$ & 0.1251 \\
\hline Blood glycosylated hemoglobin (\%) & $6.63 \pm 1.73$ & $6.67 \pm 1.72$ & 0.7277 & $5.24 \pm 0.34$ & $5.20 \pm 0.36$ & 0.1464 \\
\hline Hypercholesterolemia (\%) & 27.1 & 28.1 & 0.6220 & 29.0 & 28.8 & 0.9396 \\
\hline Serum creatinine $(\mu \mathrm{mol} / \mathrm{l})$ & $124.5 \pm 126.3$ & $62.3 \pm 12.6$ & $<0.0001$ & $89.1 \pm 23.3$ & $61.3 \pm 12.0$ & $<0.0001$ \\
\hline eGFR $\left(\mathrm{ml} / \mathrm{min} / 1.73 \mathrm{~m}^{2}\right)$ & $46.1 \pm 12.4$ & $80.4 \pm 16.4$ & $<0.0001$ & $51.5 \pm 7.2$ & $78.4 \pm 18.0$ & $<0.0001$ \\
\hline
\end{tabular}

Quantitative data are the means \pm SD. eGFR, estimated glomerular filtration rate.

Table IV. Genotype distributions of rs6929846 significantly associated with chronic kidney disease (CKD) among individuals with or without hypertension or diabetes mellitus as determined by the Chi-square test.

\begin{tabular}{|c|c|c|c|c|}
\hline Gene & SNP & CKD $(\%)$ & Controls $(\%)$ & P-value \\
\hline \multicolumn{5}{|c|}{ Without hypertension } \\
\hline \multirow[t]{4}{*}{$B T N 2 A 1$} & $\mathrm{C} \rightarrow \mathrm{T}$ & & & 0.0057 \\
\hline & $C C$ & $528(84.2)$ & $1,789(88.9)$ & \\
\hline & $C T$ & $93(14.8)$ & $215(10.7)$ & \\
\hline & $T T$ & $6(1.0)$ & $8(0.4)$ & \\
\hline \multicolumn{5}{|c|}{ With diabetes mellitus } \\
\hline \multirow[t]{4}{*}{$B T N 2 A 1$} & $\mathrm{C} \rightarrow \mathrm{T}$ & & & 0.0159 \\
\hline & $C C$ & $716(79.5)$ & $1,498(83.5)$ & \\
\hline & $C T$ & $174(19.3)$ & $286(15.9)$ & \\
\hline & $T T$ & $11(1.2)$ & $10(0.6)$ & \\
\hline \multicolumn{5}{|c|}{ With hypertension and diabetes mellitus } \\
\hline \multirow[t]{4}{*}{$B T N 2 A 1$} & $\mathrm{C} \rightarrow \mathrm{T}$ & & & 0.0488 \\
\hline & $C C$ & $570(78.8)$ & $1,075(82.6)$ & \\
\hline & $C T$ & $144(19.9)$ & $219(16.8)$ & \\
\hline & $T T$ & $9(1.3)$ & $7(0.6)$ & \\
\hline \multicolumn{5}{|c|}{ Without hypertension or diabetes mellitus } \\
\hline \multirow[t]{4}{*}{$B T N 2 A 1$} & $\mathrm{C} \rightarrow \mathrm{T}$ & & & 0.0139 \\
\hline & $C C$ & $382(85.1)$ & $1,366(89.9)$ & \\
\hline & $C T$ & $63(14.0)$ & $148(9.7)$ & \\
\hline & $T T$ & $4(0.9)$ & $5(0.4)$ & \\
\hline
\end{tabular}

and additive 1 models), in diabetic individuals (dominant and additive 1 models) and in individuals with hypertension and diabetes mellitus (dominant model), or without either condition (dominant and additive 1 models), with the $T$ allele representing a risk factor for CKD (Table V).

A stepwise forward selection procedure was performed to examine the effects of genotype for rs6929846 of BTN2AI as well as of age, gender, BMI, smoking and the prevalence of hypertension, diabetes mellitus or hypercholesterolemia on CKD (Table VI). For normotensive individuals, age, BMI, BTN2A1 genotype (dominant model) and male gender, in descending order of statistical significance, were significant $(\mathrm{P}<0.05)$ and independent determinants of CKD. For diabetic individuals, age, hypertension, BTN2Al genotype (dominant model) and male gender were significant and independent determinants of CKD. For individuals with hypertension and 
Table VI. Effects of genotype and other characteristics on chronic kidney disease among individuals with or without hypertension or diabetes mellitus determined by a stepwise forward selection procedure $(\mathrm{P}<0.05)$.

\begin{tabular}{lrc}
\hline Variable & $\mathrm{P}$-value & $\mathrm{R}^{2}$ \\
\hline Without hypertension & & \\
Age & $<0.0001$ & 0.0694 \\
BMI & 0.0002 & 0.0048 \\
BTN2AlC $(C T+T T$ vs. $C C)$ & 0.0010 & 0.0037 \\
Male gender & 0.0152 & 0.0021 \\
With diabetes mellitus & & \\
Age & $<0.0001$ & 0.0228 \\
Hypertension & $<0.0001$ & 0.0050 \\
BTN2AlC $(C T+T T$ vs. $C C)$ & 0.0105 & 0.0019 \\
Male gender & 0.0242 & 0.0015
\end{tabular}

With hypertension and

diabetes mellitus

Age

$<0.0001$

0.0185

Male gender

0.0312

0.0018

$B T N 2 A l C(C T+T T$ vs. $C C)$

0.0370

0.0016

Smoking

0.0423

0.0016

Without hypertension or

diabetes mellitus

$\begin{array}{lrr}\text { Age } & <0.0001 & 0.0818 \\ \text { BMI } & 0.0040 & 0.0039 \\ \text { BTN2AIC }(C T+\text { TT } \text { vs. CC }) & 0.0052 & 0.0037 \\ \text { Male gender } & 0.0169 & 0.0027 \\ \text { Smoking } & 0.0349 & 0.0021\end{array}$

A stepwise forward selection procedure was performed to examine the effects on CKD of the genotype for rs6929846 of BTN2Al as well as of age, gender, body mass index (BMI), smoking and the prevalence of diabetes mellitus and hypercholesterolemia in normotensive individuals; of age, gender, BMI, smoking and the prevalence of hypertension and hypercholesterolemia in diabetic individuals; of age, gender, BMI, smoking and the prevalence of hypercholesterolemia in individuals with hypertension and diabetes mellitus, or without either condition. $\mathrm{R}^{2}$, contribution rate.

diabetes mellitus, age, male gender, BTN2Al genotype (dominant model) and smoking were significant and independent determinants of CKD. For individuals without hypertension or diabetes mellitus, age, BMI, BTN2Al genotype (dominant model), male gender and smoking were significant and independent determinants of CKD.

\section{Discussion}

We examined the possible relation of rs6929846 of BTN2Al to the prevalence of CKD in Japanese individuals with or without hypertension or diabetes mellitus. Our association study with three steps of analysis (Chi-square test, multivariable logistic regression analysis with adjustment for covariates and stepwise forward selection procedure) revealed that the $\mathrm{C} \rightarrow \mathrm{T}$ polymorphism (rs6929846) of BTN2Al was significantly associated with CKD in normotensive individuals, in diabetic individuals and in individuals with hypertension and diabetes 
mellitus, or without either condition. Prediction of the risk for CKD on the basis of genetic variants is useful to decide how aggressively to target the clinical risk factors that are currently amenable to treatment.

The butyrophilin, subfamily 2 , member A1 gene (BTN2Al) is a member of the BTN2 subfamily of genes, which encode proteins belonging to the butyrophilin protein family. While the butyrophilin family was originally identified by the ability to aid production of milk fat globules (21), many butyrophilin and butyrophilin-like family of proteins were shown to regulate immune function, and polymorphisms in the coding sequences were related to predisposition to inflammatory diseases (22). Genomic mapping of disease loci identified a SNP (rs2076530) in the butyrophilin-like 2 gene (BTNL2) that increases the risk for sarcoidosis (23). Polymorphisms in BTNL2 in linkage disequilibrium with HLA-DRB were related to other inflammatory diseases, all of which can be characterized by inappropriate T-cell activation (24-26). Our previous study (16) showed that the $T$ allele increased the transcription activity of $B T N 2 A 1$ and the overexpression of BTN2Al decreased the expression of elastin mRNA and increased the mRNA expression of matrix metallopeptidase 3 and interleukin 5. These observations suggest that the $T$ allele of rs6929846 of BTN2AI may accelerate vascular inflammation (16).

The development of CKD is affected by various factors, including conventional risk factors, such as hypertension and diabetes mellitus, as well as oxidative stress and vascular inflammation. Dyslipidemia, that frequently coexists with renal disease, has been shown as an important factor in nephron damage (27). Large artery stiffness may also be related to the progression of CKD (28). We have now shown that the $\mathrm{C} \rightarrow \mathrm{T}$ polymorphism (rs6929846) of BTN2Al is significantly associated with CKD in Japanese normotensive individuals, in individuals with hypertension and diabetes mellitus, or without either condition. The enhancement of vascular inflammation by the $T$ allele of rs6929846 may account for its association with CKD, although the underlying molecular mechanisms remain unclear.

In conclusion, our results suggest that genetic variants that confer susceptibility to CKD differ among individuals with or without hypertension or diabetes mellitus. Stratification of subjects based on hypertension or diabetes mellitus may thus be important in order to achieve personalized prevention of CKD with the use of genetic information. Validation of our findings will require their replication with independent subject panels of other ethnic groups, as well as long-term follow-up to examine the association of rs6929846 of BTN2Al with the prevalence of CKD.

\section{Acknowledgements}

This study was supported in part by Grants-in-Aid for Scientific Research from the Ministry of Education, Culture, Sports, Science, and Technology of Japan (nos. 18209023, 18018021 and 19659149 to Y.Y.).

\section{References}

1. Go AS, Chertow GM, Fan D, McCulloch CE and Hsu CY: Chronic kidney disease and the risks of death, cardiovascular events, and hospitalization. N Engl J Med 351: 1296-1305, 2004.

2. Ruilope LM, Salvetti A, Jamerson K, et al: Renal function and intensive lowering of blood pressure in hypertensive participants of the hypertension optimal treatment (HOT) study. J Am Soc Nephrol 12: 218-225, 2001.

3. National Kidney Foundation: K/DOQI clinical practice guidelines for chronic kidney disease: evaluation, classification, and stratification. Am J Kidney Dis 39 (Suppl 1): 1-266, 2002.

4. Yamagata K, Ishida K, Sairenchi T, et al: Risk factors for chronic kidney disease in a community-based population: a 10-year follow-up study. Kidney Int 71: 159-166, 2007.

5. Norris KC, Tareen N, Martins D and Vaziri ND: Implications of ethnicity for the treatment of hypertensive kidney disease, with an emphasis on African Americans. Nat Clin Pract Nephrol 4: 538-549, 2008.

6. Sarafidis PA, Li S, Chen SC, et al: Hypertension awareness, treatment, and control in chronic kidney disease. Am J Med 121: 332-340, 2008

7. James MT, Hemmelgarn BR and Tonelli M: Early recognition and prevention of chronic kidney disease. Lancet 375: 1296-1309, 2010.

8. Yoshida T, Kato K, Yokoi K, et al: Association of candidate gene polymorphisms with chronic kidney disease in Japanese individuals with hypertension. Hypertens Res 32: 411-418, 2009.

9. Kottgen A, Kao WH, Hwang SJ, et al: Genome-wide association study for renal traits in the Framingham Heart and Atherosclerosis Risk in Communities Studies. BMC Med Genet 9: 49, 2008

10. Chambers JC, Zhang W, Lord GM, et al: Genetic loci influencing kidney function and chronic kidney disease. Nat Genet 42: 373-375, 2010.

11. Köttgen A, Pattaro C, Böger CA, et al: New loci associated with kidney function and chronic kidney disease. Nat Genet 42: 376-384, 2010.

12. Köttgen A, Glazer NL, Dehghan A, et al: Multiple loci associated with indices of renal function and chronic kidney disease. Nat Genet 41: 712-717, 2009.

13. Kao WH, Klag MJ, Meoni LA, et al; Family Investigation of Nephropathy and Diabetes Research Group: MYH9 is associated with nondiabetic end-stage renal disease in African Americans. Nat Genet 40: 1185-1192, 2008.

14. Gharavi AG, Yan Y, Scolari F, et al: IgA nephropathy, the most common cause of glomerulonephritis, is linked to 6q22-23. Nat Genet 26: 354-357, 2000.

15. Hanson RL, Craig DW, Millis MP, et al: Identification of PVT1 as a candidate gene for end-stage renal disease in type 2 diabetes using a pooling-based genome-wide single nucleotide polymorphism association study. Diabetes 56: 975-983, 2007.

16. Yamada Y, Nishida T, Ichihara S, et al: Association of a polymorphism of BTN2A1 with myocardial infarction in East Asian populations. Atherosclerosis (In Press).

17. Yoshida T, Kato K, Fujimaki T, et al: Association of a polymorphism of the apolipoprotein E gene with chronic kidney disease in Japanese individuals with metabolic syndrome. Genomics 93: 221-226, 2009.

18. Yoshida T, Kato K, Yokoi K, et al: Association of genetic variants with chronic kidney disease in Japanese individuals with type 2 diabetes mellitus. Int J Mol Med 23: 529-537, 2009.

19. Yoshida T, Kato K, Yokoi K, et al: Association of genetic variants with chronic kidney disease in individuals with different lipid profiles. Int J Mol Med 24: 233-246, 2009.

20. Matsuo S, Imai E, Horio M, et al: Revised equations for estimated GFR from serum creatinine in Japan. Am J Kidney Dis 53: 982-992, 2009.

21. Ogg SL, Weldon AK, Dobbie L, Smith AJ and Mather IH: Expression of butyrophilin (Btn1a1) in lactating mammary gland is essential for the regulated secretion of milk-lipid droplets. Proc Natl Acad Sci USA 101: 10084-10089, 2004.

22. Arnett HA, Escobar SS and Viney JL: Regulation of costimulation in the era of butyrophilins. Cytokine 46: 370-375, 2009.

23. Valentonyte R, Hampe J, Huse K, et al: Sarcoidosis is associated with a truncating splice site mutation in BTNL2. Nat Genet 37 : 357-364, 2005. 
24. Mochida A, Kinouchi Y, Negoro K, et al: Butyrophilin-like 2 gene is associated with ulcerative colitis in the Japanese under strong linkage disequilibrium with HLA-DRB1*1502. Tissue Antigens 70: 128-135, 2007.

25. Orozco G, Eerligh P, Sánchez E, et al: Analysis of a functional BTNL2 polymorphism in type 1 diabetes, rheumatoid arthritis, and systemic lupus erythematosus. Hum Immunol 66: 1235-1241, 2005 .

26. Traherne JA, Barcellos LF, Sawcer SJ, et al: Association of the truncating splice site mutation in BTNL2 with multiple sclerosis is secondary to HLA-DRB1*15. Hum Mol Genet 15: 155-161, 2006 .
27. Keane WF, Kasiske BL, O'Donnell MP and Kim Y: Hypertension, hyperlipidemia, and renal damage. Am J Kidney Dis 21 (Suppl 2): 43-50, 1993.

28. Fassett RG, D'Intini V, Healy $\mathrm{H}$, et al: Assessment of arterial stiffness, oxidative stress and inflammation in acute kidney injury. BMC Nephrol 10: 15, 2009. 